Mario Biagioni

Pistoia - Independent Scholar

\title{
Fonti umanistiche nel pensiero di Christian Francken ${ }^{1}$
}

Radykalny reformator Christian Francken (1552-1610?) pozostawał pod ogromnym wpływem Pietra Pomponazziego, a później Sekstusa Empiryka. Znacznie mniej istotny był wpływ neoplatonizmu. Jego wczesne teologiczne i filozoficzne teksty, od Breve colloquium Iesuiticum (1579) do Praecipuarum enumeratio causarum (1584), czerpały przede wszystkim z prac Pomponazziego. Wpływ Sekstusa Empiryka stał się fundamentalny około 1587 r., kiedy Francken zaczął pracę nad dziełem Disputatio de incertitudine religionis Christianae, w którym ujawnił zdecydowanie sceptyczną postawę, graniczącą $\mathrm{z}$ niewiarą.

The radical reformer Christian Francken (1552-1610?) was deeply influenced by Pietro Pomponazzi and, later, by Sextus Empiricus. Much less important was the influence of Neoplatonism. Pomponazzi's works were the main sources in his early theological and philosophical writings from the Breve colloquium Iesuiticum (1579) to the Praecipuarum enumeratio causarum (1584). The influence of Sextus Empiricus became crucial from

${ }^{1}$ Il presente saggio presuppone le tre seguenti opere, alle quali si rimanda per le necessarie conoscenze biografiche e bibliografiche: L. Szczucki, Philosophie und Autorität. Der Fall Christian Francken, in: Reformation und Frühaufklärung in Polen. Studien über den Sozinianismus und seine Einflu $\beta$ auf das westeuropäische Denken im 17. Jahrhundert, hrsg. von P. Wrzecionko, Gottingen 1977, pp. 157-243; J. Simon, Die Religionsphilosophie Christian Franckens 1552-1610? Atheismus und radikale Reformation in Frühneuzeitlichen Ostmitteleuropa, Wiesbaden 2008; M. Biagioni, Christian Francken e la crisi intellettuale della Riforma, in: Ch. Francken, Opere a stampa, a cura di M. Biagioni, premessa di G. Paganini, Roma 2014, pp. 3-150. 
around 1587, when Francken began writing the Disputatio de incertitudine religionis Christianae, where he exhibited a sharp skeptical attitude that led him not far from unbelief.

Ke yw o rds: radykalna reformacja, sceptycyzm, Sekstus Empiryk, Pomponazzi, Francken, niewiara

Ke yw o rds: Radical Reformation, skepticism, Sextus Empiricus, Pomponazzi, Francken, unbelief

Uno dei problemi storiografici più ardui per gli studiosi della Riforma radicale consiste nella ricerca delle fonti che contribuirono alla formazione e poi alla costruzione del percorso intellettuale di uomini che, nella quasi totalità, vennero marginalizzati e non entrarono a fare parte di istituzioni, di comunità organizzate, di gruppi ufficialmente riconosciuti. La loro principale caratteristica fu la mobilità, la refrattarietà verso classificazioni e collocazioni definitive. Si tratta di personaggi sconfitti dalla storia, costretti a nascondersi, a mimetizzarsi, a dissimulare per sopravvivere, e non solo all'interno del mondo cattolico a causa della forza coercitiva dell'inquisizione, ma anche nell'Europa protestante, dove almeno a partire dalla metà del Cinquecento il processo di confessionalizzazione iniziò a restringere progressivamente gli spazi concessi alla libertà religiosa. Spesso i loro percorsi intellettuali risultarono trasversali rispetto ai tradizionali centri di formazione (conventi, collegi ecclesiastici, università) e si arricchirono con esperienze di lettura favorite dall'ampia circolazione di libri a stampa, con la frequentazione di gruppi clandestini o comunque riuniti in contesti privati, e soprattutto grazie alla fittissima rete di relazioni interpersonali, di incontri che favorivano la discussione orale delle idee e potevano rivelarsi decisivi. Ancora nel Cinquecento, del resto, la memorizzazione di testi o di parti importanti di essi suppliva molte volte al possesso materiale dei libri. Non era una circostanza straordinaria, dunque, quella che ricordava Tommaso Campanella nel Quod reminiscentur quando attribuiva a Francesco Pucci, incontrato nel carcere romano del Santo Uffizio, la capacità di recitare a memoria le opere di Lutero e di Calvino ${ }^{2}$. Neppure stupisce che il magistero di Juan de Valdés a Napoli e successivamente la diffusione del suo pensiero, come ha dimostrato Massimo Firpo, si fondassero soprattutto sulla

${ }^{2}$ T. Campanella, Quod reminiscentur et convertentur ad dominum universi fines terrae, a cura di R. Amerio, vol. 1, Padova 1939, p. 144. 
circolazione orale delle idee, sui contatti diretti tra coloro che avevano apprezzato la sua dottrina, tutt'al più sul passaggio di mano in mano di alcune carte manoscritte, secondo meccanismi di trasmissione che recuperavano dinamiche proprie della realtà precedente all'affermazione della stampa ${ }^{3}$. Entro una simile cornice, appare evidente quale sia la complessità di ogni tentativo di mappatura delle fonti che si pongono alla base di sistemi di pensiero così mutevoli, schermati dalla reticenza e sviluppati seguendo percorsi alternativi rispetto alle vie tradizionali. Il poderoso tentativo messo in atto nel 1957 da George Huntston Williams di classificare tutti i movimenti e i personaggi di quel fenomeno da lui stesso denominato Riforma Radicale, se da un lato ha rivelato i limiti derivanti dall'adozione di criteri squisitamente teologici per individuare uomini e gruppi per loro natura riluttanti a inoppugnabili confessiones fidei proprio in difesa della libertà di pensiero, dall'altro ha reso palese la straordinaria varietà di sfumature nella quale si sfrangiava questa categoria storica quanto più si scendeva addentro ai dettagli del quadro $^{4}$. Anche la grande suddivisione da lui proposta in sole tre aree di pensiero, gli anabattisti, gli spiritualisti e i razionalisti evangelici (tra i quali i sociniani), suscitò un vivace dibattito storiografico, che toccava il delicato e fondamentale problema di che cosa si intendesse con il termine radicalismo in relazione alla Riforma ${ }^{5}$. Entro un orizzonte così in espansione, anche la nozione cantimoriana di „eretici italiani del Cinquecento”, che aveva dato il titolo al celebre libro del 1939, mostrava alcuni limiti, aprendo spazi per una revisione alla quale lo stesso Cantimori iniziò a lavorare negli ultimi anni della sua vita. Ne rendeva conto nelle Prospettive di storia

${ }^{3}$ Cito soltanto la sua più recente pubblicazione, alla quale rimando per una bibliografia completa: M. Firpo, Juan de Valdés e la Riforma nell'Italia del Cinquecento, Roma-Bari 2016 (anche in edizione inglese: M. Firpo, Juan de Valdés and the Italian Reformation, Ashgate 2015).

${ }^{4}$ G. H. Williams, The Radical Reformation, Philadelphia 1962 ( $1^{\circ}$ ed.). La terza edizione dell'opera (Kirksville 2000) conta circa 600 pagine in più rispetto alla prima. Osservazioni circa la difficoltà di classificare i numerosi esuli religionis causa in: A. Rotondò, I movimenti ereticali nell'Europa del Cinquecento. Discussione storiografica, „Rivista Storica Italiana”, LXXVIII, 1966, pp. 103-139, ora in: idem, Studi di storia ereticale del Cinquecento, vol. 1, Firenze 2008, pp. 1-43.

${ }^{5}$ Vedi J. D. Roth, Recent Currents in the Historiography of the Radical Reformation, "Church History”, 71/ 3, 2002, pp. 523-535; M. Biagioni, The Radical Reformation and the Making of Modern Europe. A Lasting Heritage, Leiden-Boston 2017, pp. 7-26. 
ereticale italiana del Cinquecento, uscito nel $1960^{6}$, dove proponeva nuovi temi, irrobustiva alcune delle coordinate utilizzate in precedenza, cercava di superare quell'ottica degli italiani all'estero, esportatori di una cultura umanistica autoctona e refrattaria a qualunque tipo di confessionalizzazione, che aveva presieduto all'iniziale progetto di ricerca ${ }^{7}$.

Proprio a questa iniziale prospettiva cantimoriana Luigi Firpo aveva collegato, in un suo importante studio del 1958, anche il personaggio di Christian Francken, nonostante si trattasse di „un autentico prussiano", per usare le sue parole. L'operazione, non priva di qualche passaggio acrobatico, si fondava sulla considerazione che comunque Francken aveva studiato nel Collegio Romano e aveva avuto rapporti con molti esuli italiani religionis causa, potendo così attingere alle stesse sorgenti italiche di cultura umanistica ${ }^{8}$. Si tratta oggi di una chiave di lettura difficilmente sostenibile nel suo complesso, ma non priva di elementi di sicuro interesse, soprattutto perché fondata su un apparato documentario che, come sempre nell'attività di Luigi Firpo, attingeva a una erudizione profonda. Gli studi successivi, in realtà, hanno dimostrato quanta parte abbiano avuto nel modellare il pensiero di Francken le esperienze maturate nei paesi della Riforma, il rapporto con gli antitrinitari Polacchi e Transilvani, la scoperta dei testi di Sesto Empirico, consentendo così di leggere la giravolta delle sue conversioni sotto una luce diversa, rispetto al tranciante giudizio di un uomo ,venato da un ramo di inquieta stravaganza, per non dir di follia". Ma è un fatto che nella prima opera a stampa, il Breve colloquium Iesuiticum, pubblicata a Lipsia nel $1579^{10}$, egli esprime un pensiero che appare solidamente incardinato

${ }^{6}$ Ora in: D. Cantimori, Eretici italiani del Cinquecento e prospettive di storia ereticale italiana del Cinquecento, a cura di A. Prosperi, Torino 1992, pp. 419-481.

7 Vedi A. Rotondò, Alcune valutazioni su „Eretici italiani del Cinquecento”, „Studi Storici”, 34/4, 1993, pp. 769-775.

${ }^{8}$ L. Firpo, Il vero autore di un celebre scritto antitrinitario: Christian Francken e non Lelio Socino, „Bollettino della Società di Studi Valdesi”, LXXXVII, 1958, pp. 51-68: 52, ora in: idem, Scritti sulla Riforma in Italia, Napoli 1996, pp. 96-115: 97. Sempre di L. Firpo, vedi anche Christian Francken antitrinitario, „Bollettino della Società di Studi Valdesi”, LXXVIII, 1960, pp. 27-35 (ora in: idem, Scritti, pp. 195-204).

${ }^{9}$ L. Firpo, Scritti, p. 97. Una interpretazione in chiave scettica delle numerose conversioni di Francken in Gianni Paganini, Scetticismo e religione in Christian Francken, in: C. Francken, op. cit., pp. IX-XIX.

${ }^{10}$ Ora in: C. Francken, op. cit., pp. 157-177. 
sull'aristotelismo acquisito negli anni di studio presso il Collegio romano. Del resto, non avendo notizie precedenti al suo ingresso nella Compagnia di Gesù a Vienna nel 1568 all'età di soli sedici anni, la storia della formazione di Francken inizia per gli studiosi con il trasferimento a Roma, così come indicato da Firpo. Degli insegnanti che lo seguirono negli studi al Collegio romano restano alcune testimonianze nelle opere successive al Colloquium, ma per lo più filtrate attraverso lo spirito polemico della diatriba contro la Compagnia di Gesù. Tra questi Iacobus Paetz, nominato nel De bestialissima idololatria, che uscì a Basilea nel 1580, contro la dottrina cattolica della transustanziazione ${ }^{11}$. Paetz insegnò teologia scolastica al Collegio romano dal 1565 al 1576, e Francken lo ricorda ironicamente per avere acquistato fama grazie a un unico motivo, cioè la critica alle perplessità espresse dal cardinale Tommaso De Vio nel commento alla Summa di Tommaso d'Aquino circa la presenza reale del corpo e del sangue di Cristo nell'eucaristia ${ }^{12}$, un atteggiamento che naturalmente Francken tratta con dileggio. Sempre in chiave negativa, anche se in un contesto più articolato, tornano i nomi di Benedetto Pererira e Francisco De Toledo nella Praecipuarum enumeratio causarum del 1584, opera di feroce critica al dogma trinitario ${ }^{13}$. Benedetto Pereira, filosofo aristotelico che legò il suo nome al $D e$ communibus omnium rerum naturalium principiis del $1576^{14}$, passò dall'insegnamento di Metafisica a quello di Teologia scolastica nel 1567 , anche a causa di tendenze platonizzanti giudicate con qualche sospetto, e infine ricoprì la cattedra di Sacra Scrittura dal 1570 in poi ${ }^{15}$. Francisco De Toledo fu una figura di assoluto rilievo nella Chiesa romana di fine secolo (prese le difese di Bartolomeo Carranza durante il processo, venne inviato a Lovanio a raccogliere la ritrattazione di Pietro Baio), ma il suo insegnamento di Teologia scolastica nel Collegio romano creò aspri dissensi con alcuni

${ }^{11}$ Ora ibidem, pp. 179-189.

12 Ibidem, p. 180.

13 Ora ibidem, pp. 243-258. Precedentemente pubblicata in: L. Szczucki, W kregu myślicieli heretyckich, Wrocław 1972, pp. 256-265.

${ }^{14}$ Benedicti Pererii Societatis Iesu De communibus omnium rerum naturalium principiis et affectionibus libri quindecim qui plurimum conferunt ad eos octo libros Aristotelis qui de physico auditu inscribuntur intelligendos, apud Franciscum Zanettum et Bartholomaeum Tosium, Romae 1576.

15 Vedi R. G. Villoslada, Storia del collegio romano dal suo inizio (1551) alla soppressione della Compagnia di Gesù (1773), Romae 1954, pp. 51-52. 
colleghi, soprattutto riguardo alla dottrina della predestinazione, tanto da spingerli a una denuncia presso il generale Lainez ${ }^{16}$. Francken in realtà, a proposito di Francisco da Toledo, cita non uno scritto teologico ma un commento ad Aristotele dal titolo Commentaria una cum quaestionibus in octo libris Aristotelis de physica auscultatione uscito a Venezia nel 1573, laddove discute della creazione della materia in relazione al concetto di coeternità ${ }^{17}$. Echi delle esperienze acquisite negli anni di formazione a Roma ritornano in vari luoghi dell'opera di Francken in forme più o meno esplicite. Piuttosto chiara è, per esempio, la scarsa stima nei confronti di Pietro Maffei, che ricoprì a più riprese la cattedra di Retorica, e che Francken tende addirittura a ridicolizzare per la sua biografia di Ignazio di Loiola pubblicata a Venezia nel 1585 e da lui ritenuta un degno esempio della credulità, falsità e idolatria gesuitica ${ }^{18}$. Possiamo forse congetturare che anche il ricorso all'immagine della forza cogente che deriva dalle leggi della matematica contrapposta alle conclusioni solo probabili della teologia, con la quale si apre la Disputatio de incertitudine religionis Christianae del $1593^{19}$, abbia radici lontane nel dibattito che si svolse nelle aule del Collegio romano, per poi affidarsi alla carta stampata, tra Cristoforo Clavio, docente di Matematica e Astronomia dal 1564 al 1571 e poi dal 1576 al 1584, nonché amico di Galilei, e il già citato Benedetto Pereira. Come è noto, quest'ultimo sosteneva che la matematica non poteva avere carattere dimostrativo dei fenomeni naturali e quindi non era vera scienza, schierandosi con coloro che ritenevano inopportuno impartire tale insegnamento nei collegi gesuitici. La questione della certezza della matematica aveva assunto toni particolarmente accesi all'interno della Compagnia e alla sua discussione Clavio fornì un contributo decisivo con alcuni scritti, tra i quali soprattutto gli In disciplinas mathematicas prolegomena del 1574, premessi al commento sugli Euclidis Elementa ${ }^{20}$. Alla fine la sua

${ }^{16}$ Ibidem, pp. 75-76.

17 C. Francken, op. cit., p. 249.

18 G. P. Maffei, De vita et moribus Ignatii Loyolae, qui Societatem Iesu fundavit, libri III, apud Franciscum Zannettum, Romae 1585. Vedi M. Biagioni, Christian Francken, pp. 124-127. L'opera è ora pubblicata in: C. Francken, op. cit., pp. 263-271.

19 J. Simon, op. cit., p. 153.

20 Christophorus Clavius Bambergensis Euclidis elementorum libri XV, apud Vincentium Accoltum, Romae 1574. Vedi R. Gatto, Cristoforo Clavio, in: Enciclopedia Italiana di Scienze, Lettere ed Arti. Contributo italiano alla storia del pensiero. Ottava appendice, Roma 2013, pp. 236-240. 
battaglia ottenne successo con l'inserimento della matematica nella Ratio studiorum pubblicata nel 1586.

Al di là di questi richiami a episodi della formazione di Francken, l'intero impianto del Breve colloquium Iesuiticum appare calato in una prospettiva che coincide con quella dell'aristotelismo imperante nel Collegio romano. Lech Szczucki ha suggerito una fonte aristotelica per la concezione della dipendenza degli uomini dalle inclinazioni naturali ${ }^{21}$, alla quale viene concesso molto spazio nell'opera, naturalmente declinata in chiave antigesuitica ${ }^{22}$. Sebbene a utilizzarla sia il personaggio di Paul Florenius, amico e interlocutore di Francken nel dialogo che si svolge durante una pausa delle attività del Collegio, essa viene ripresa e fatta propria anche dall'autore cinque anni dopo nella Praecipuarum enumeratio causarum ${ }^{23}$. Nel primo libro della Politica, discutendo dell'organizzazione della famiglia, Aristotele distingue tra padroni e schiavi sostenendo la loro naturale diversità, che privilegia nei primi la facoltà di governare, negli altri quella di obbedire, secondo un rapporto che riproduce quello tra anima e corpo ${ }^{24}$. Nel Breve colloquium Francken estende l'idea della forza delle inclinazioni naturali proponendo un vero e proprio sistema dei principali tipi umani e riconoscendo quattro gruppi: ispanici, italici, gallici e germanici. Nella Compagnia di Gesù prevalgono i primi due per la tendenza degli uni alla cieca obbedienza, degli altri alla superficialità e alla vanagloria, e tra di essi hanno la meglio gli stolti e i servili rispetto agli ingegnosi e agli onesti: sono loro a spartirsi le più alte cariche e a ricevere le maggiori attestazioni di riverenza e di santità ${ }^{25}$. $\mathrm{Ma}$ a prescindere da questo esito particolare, è lo stretto naturalismo dal quale esso deriva che rappresenta il tratto dominante del pensiero di Francken in questa prima fase e che si pone alla base della crisi religiosa che lo spinse a lasciare la Compagnia di Gesù. Egli sembra volere raccogliere la sfida dell'apparente inconciliabilità tra insegnamento teologico e filosofia aristotelica, aperta già all'interno della cultura scolastica medievale, tenendo però come punto fermo la verità filosofica. Si tratta di un atteggiamento che lo porterà ad alcune consonanze con l'aristotelismo radicale di Pietro Pomponazzi. In realtà

${ }^{21}$ L. Szczucki, Philosophie, pp. 169-170.

${ }^{22}$ C. Francken, op. cit., pp. 164-167.

${ }^{23}$ Ibidem, p. 244.

${ }^{24}$ Aristotele, Politica, I, 4-5.

${ }^{25}$ C. Francken, op. cit., p. 165. Oltre al già citato L. Szczucki (Philosophie, p. 170). Vedi anche M. Biagioni, Christian Francken, pp. 29-30. 
il nome di Pomponazzi compare solo nell'ultima opera di Francken, l'Analysis rixae Christianae del $1595^{26}$, ma l'affinità di idee lascia credere a una conoscenza precedente e decisiva nella sua evoluzione intellettuale. La formazione filosofica ricevuta a Roma era quasi certamente estranea a simili forme di radicalismo, ma tale da innescare comunque nella mente inquieta del giovane sassone, incapace di scendere a compromessi, interrogativi profondi che egli non sapeva lasciare senza risposta. Una delle immagini che più restano impresse, tra le tante che animano le pagine del Breve colloquium, è probabilmente quella dell'angelo caduto, con la quale si apre l'ultimo, esteso intervento di Francken nel dialogo con Florenius ${ }^{27}$. Francken era entrato nella Compagnia di Gesù all'età di sedici anni e si era dedicato allo studio e alle pratiche devozionali con la ferma volontà di un giovane entusiasta, convinto di avere intrapreso la strada che porta verso il cielo. Egli giudicava divina la Compagnia e sentiva che „non amplius in terris, sed versari videbar in coelis”: era nato di nuovo, questa volta non da genitori terreni, ma da Cristo stesso, tanto da definirsi „velut iniesuanatus, non aliter ac Christus a spiritu sancto incarnatus" 28 . Improvvisamente, però, dopo anni di privazioni che ne avevano indebolito il corpo, leggendo il Commentarius de rebus Indicis et Iaponicis di Manoel da Costa che riferiva di popoli pagani con costumi religiosi molto simili a quelli dei gesuiti ${ }^{29} \mathrm{e}$, soprattutto, riflettendo sulle parole dell'antico testamento, in particolare $1 \mathrm{Re}$ XVIII, 28 dove si descrivono le pene corporali che i sacerdoti di Baal erano soliti infliggersi ${ }^{30}$, anch'esse analoghe ai sacrifici praticati nella Compagnia, egli era precipitato nel baratro del dubbio, prima stupito, poi inorridito, come „si ex astris in terram relapsus fuissem” 31 . Francken aveva circa venticinque anni e per la prima volta nella sua mente prendeva corpo la convinzione che la corrispondenza tra il culto di popoli idolatri e quello della Compagnia di Gesù costituisse una prova indubitabile dell'origine terrena anche di quest'ultima. Si trattava del motivo che lo spinse all'estenuante ricerca di un criterio di verità in grado di separare nella religione ciò che viene dal mondo

\footnotetext{
${ }^{26}$ C. Francken, op. cit., p. 289.

${ }^{27}$ Ibidem, p. 171.

${ }^{28}$ Ibidem, p. 170.

${ }^{29}$ Si tratta di Manoel da Costa, Rerum e Societate Iesu in Oriente gestarum volumen, Calenius et Quentel, Colonia 1574.

${ }^{30}$ C. Francken, op. cit., p. 173.

${ }^{31}$ Ibidem, p. 171.
} 
e ciò che viene dal cielo, costringendolo a un cammino tortuoso in tutte le chiese della Riforma, sino al drammatico esito del processo romano presso il Santo Uffizio e della condanna al carcere a vita nel $1601^{32}$. Per quanto concerne il problema delle fonti, il dato che colpisce di più è rappresentato dalla lettura in chiave rigorosamente naturalistica della corrispondenza tra i culti di popoli posti agli antipodi. Rimane al di fuori dell'orizzonte di Francken qualunque ipotesi innatistica di derivazione platonica, che renda plausibile considerarla una prova dell'esistenza nella mente di tutti gli uomini dell'idea innata di Dio. Egli esclude quindi la possibilità di una religione naturale e non prende affatto in considerazione l'argomento del consensus gentium $^{33}$, sebbene esso fosse presente in Aristotele ${ }^{34} \mathrm{e}$, attraverso l'alveo dello stoicismo e dell'eclettismo ellenistico, fosse confluito nell'opera di Cicerone, del quale pure nel Breve colloquium sono citate anche le Tusculanae, dove il tema viene affrontato ${ }^{35}$. Non emergono neppure precisi richiami a Tommaso d'Aquino, sebbene esso rappresenti sicuramente un caposaldo della formazione di Francken, come si può desumere dalla presenza del suo pensiero in opere successive, oggi più chiaramente individuabile grazie all'analisi condotta da József Simon sul testo della Disputatio de incertitudine del $1593^{36}$. Certo, tutto il pensiero della Scolastica medievale costituisce un presupposto ineludibile del percorso intellettuale di Francken, con Anselmo d'Aosta, Duns Scoto e Guglielmo di Ockham avanti agli altri. Ma subito dopo l'uscita dalla Compagnia, egli lo fa coincidere soprattutto con il bersaglio della rivolta contro la cieca obbedienza alla lezione dei maestri che i gesuiti imponevano per giustificare le più assurde superstizioni,

32 Sul processo di Francken, vedi soprattutto: L. Szczucki, Philosophie, pp. 237-243; J. Wijaczka, Christian Francken, in: Bibliotheca dissidentium. Repertoire des non-conformistes religieux des seizième et dix-septième siècles, a cura di A. Séguenny, t. 12, Baden-Baden 1991, pp. 99, 108-109. A proposito dell'ultima notizia della sua esistenza in vita vedi J. Simon, op. cit., p. 10.

33 Vedi M. Biagioni, La critica all'argomento del "consensus gentium” in Christian Francken e François de La Mothe Le Vayer, in: Le ragioni degli altri, a cura di M. Priarolo, E. Scribano, Philosophica 3, Edizioni Ca' Foscari Digital Publishing, febbraio 2017, http://edizionicafoscari.unive.it/it/edizioni/collane/philosophica/, pp. 47-62.

${ }^{34}$ Per esempio in: Aristotele, Etica a Nicomaco, X, 2, 1173a.

35 M. Tullius Cicero, Tusculanae Disputationes, I, 13, 30. Oltre alle Tusculanae, sono citate anche il De natura deorum e la In Gaium Verrem actionis secundae liber IV (vedi C. Francken, op. cit., p. 176).

36 J. Simon, op. cit. 
e anche Tommaso viene quindi investito dal giudizio negativo nei confronti di tale atteggiamento ${ }^{37}$. Non è forse casuale che tra gli insegnanti che egli più tardi nomina in chiave polemica compaiano proprio Iacobus Paetz e Benedictus Pereira, entrambi docenti di teologia scolastica ${ }^{38}$.

Dal Breve colloquium e dagli altri scritti antecedenti la prima conversione al Socinianesimo, collocabile intorno al 1583, si trae complessivamente l'impressione di trovarsi dinanzi a un autore dotto, con adeguata conoscenza dei classici (oltre alle opere retoriche e filosofiche di Cicerone, sono richiamate anche quelle di Macrobio, Ovidio, Varrone $)^{39}$ e una cultura filosofica saldamente impiantata su fonti aristoteliche, forse non particolarmente originale, ma ravvivata da uno spirito inquieto e tendenzialmente propenso a radicalizzarne gli insegnamenti. In effetti, nonostante le cautele verso la Scolastica, Francken sembra rimanere all'interno di una prospettiva concordistica tra teologia cristiana e filosofia aristotelica, ma con una importante peculiarità: è la religione che non può sostenere dogmi contrari all'evidenza razionale. Tali sono i presupposti della sua lotta contro le forme idolatriche e l'assurdità dei dogmi del Cristianesimo, secondo un principio formulato nel De bestialissima idololatria, ossia che: „Theologia autem etsi ea docet quae sunt supra rationem et u'perfusika, non tamen quae contra et avntifusika" ${ }^{40}$. Se non che, senza la prospettiva di una doppia verità, ciò che si pone sopra la ragione appare inevitabilmente anche contro la ragione. In base a questo principio nell'ultima parte del Breve colloquium Iesuiticum Francken si lancia all'attacco dei due principali idoli della Chiesa di Roma: il pontefice romano e la Madonna di Loreto. La pratica degli ex voto collegata al culto di quest'ultima lo spinge a condividere le parole di Cicerone nel terzo libro del De natura deorum, laddove afferma che le immagini votive raffigurano solo chi è scampato al pericolo, non gli innumerevoli altri che invece sono deceduti ${ }^{41}$ : è la superstizione, quindi, a trasformare in un evento miracoloso quanto è frutto della semplice

37 Tommaso d'Aquino è citato in chiave polemica nel De bestialissima idololatria (C. Francken, op. cit., p. 184) e poi nel De praedestinatione (ibidem, pp. 194-195).

38 Ibidem, pp. 180, 249.

${ }^{39}$ Macrobius, Saturnalia, I, 13, 3; P. Ovidius Naso, Fastorum libri, II; M. Terentius Varro, De lingua Latina, VI, 13, 34 (vedi C. Francken, op. cit., p. 166).

${ }^{40}$ C. Francken, op. cit., pp. 183-184.

${ }^{41}$ M. Tullius Cicero, De natura deorum libri, III, 89 (vedi C. Francken, op. cit., pp. 175-176). 
casualità e a cercarne origine nella provvidenza. Si tratta di argomenti affini a quelli utilizzati da Pietro Pomponazzi nel De incantationibus, composto intorno al 1520 ma pubblicato postumo a Basilea nel $1556^{42}$. In effetti, come abbiamo detto, il problema del rapporto tra l'aristotelismo radicale del filosofo mantovano e alcune idee formulate da Francken, soprattutto a partire dal 1583, rappresenta una questione esegetica tra le più interessanti nel lavoro di scandaglio sulle fonti del suo pensiero. Lech Szczucki ${ }^{43}$ nota che uno degli argomenti da lui utilizzati per negare la plausibilità filosofica del dogma trinitario nella Praecipuarum enumeratio causarum del 1584, ossia la paradossalità della generazione $a b$ aeterno del figlio, prende spunto da una posizione contenuta nel De fato, libero arbitrio et praedestinatione di Pomponazzi $^{44}$. Seguendo una logica analoga, infatti, Francken afferma che nell'eternità, con ogni evidenza, non esiste un prima e un dopo. Quindi è stolto parlare di generazione $a b$ aeterno perché o „non fuit generatio, id est productio de non esse ad esse" o non fu dall'eternità, in quanto „cum generatus est, coepit habere esse, ergo non ab aeterno fuit" ${ }^{25}$. Se non che Francken tralascia la distinzione fondamentale tra „ex parte modi nostri intelligendi” ed „ex parte rei”, ossia tra modalità del conoscente ed essenza del conosciuto, che apriva la prospettiva di una doppia verità, per negare sic et simpliciter qualsiasi plausibilità alla concezione trinitaria, lasciando presumere una soluzione assai più drastica. È difficile stabilire con quanta consapevolezza e giovandosi di quali fonti egli intraprenda un simile percorso. La Praecipuarum enumeratio causarum fu composta in stretta correlazione con la disputa De honore Christi contro Fausto Sozzini che si svolse nel mese di marzo a Pawlikovice presso la residenza di Krzysztof Morsztyn ${ }^{46}$. Le

42 Vedi ora: P. Pomponazzi, De incantationibus, a cura di V. Perrone Compagni, con la collaborazione codicologica di L. Regnicoli, Firenze 2011.

${ }^{43}$ L. Szczucki, Philosophie, p. 204.

44 „Unde nulla cadit in Deo mutatio; quicquid enim videt, aeternaliter videt. Quanquam secundum nostrum modum intelligendi concedi potest in Deo et in suis operationibus esse prioritatem et posterioritatem; sicut dicimus Deum prius intelligere quam velle, et pro aliquo signo intelligere pro quo non est velle; sed hoc arguit novitatem ex parte modi nostri intelligendi, non autem ex parte rei", Petri Pomponatii Mantuani Libri quinque de fato, de libero arbitrio et de praedestinatione, III, 14, a cura di R. Lemay, Padova 1957, pp. 313-314.

45 C. Francken, op. cit., p. 248.

46 Pubblicata in: Fausti Socini Senensis Opera Omnia in duos tomos distincta, vol. 2, Irenopoli [Amsterdam] 1656, pp. 767-777 (vedi anche la ristampa a cura di E. Scribano, Siena 2004). Ora in: C. Francken, op. cit., pp. 213-241. 
due opere sono calate nel contesto delle dispute teologiche che divisero in quegli anni la chiesa antitrinitaria polacca, da un lato sempre più raccolta intorno alle posizioni di Fausto Sozzini, dall'altro ancora attratta da quelle più radicali del gruppo che si riconosceva nel pensiero di Ferenc Dávid e aveva il suo centro in Transilvania: Francken sosteneva a Cracovia le posizioni non adorantiste di questi ultimi. Ma nella Praecipuarum enumeratio, in realtà, l'obiettivo polemico continua ad essere rappresentato innanzi tutto dagli epigoni della tradizione scolastica, fra i quali alcuni degli insegnanti del Collegio romano esplicitamente nominati, come Benedetto Pereira e Francisco de Toledo ${ }^{47}$. Il momento della conversione di Francken all'antitrinitarismo sembra provocare oscillazioni importanti che tendono a consolidare piuttosto che a travalicare l'orizzonte dell'aristotelismo critico che aveva contraddistinto i suoi primi passi. Per esempio, l'idea dell'uso politico della religione, implicita nel Breve colloquium, viene sviluppata nella Epistola Nicolai Regi Germani composta sotto falso nome a Cracovia nel 1583 contro la concezione controriformistica della Chiesa cristiana proclamata dal cardinale Krzysztof Warszewi$\mathrm{cki}^{48}$. Francken vi afferma che fino dall'antichità molti legislatori approfittarono della credulità del volgo piegandola ai loro fini, così come oggi fa il pontefice. Tra questi Zoroastro, Zaleuco, Zalmoxis, Caronda, Solone, Licurgo, Minosse, Numa Pompilio, che fecero credere di essere stati ispirati dalle divinità nel redigere la legge in modo da incutere soggezione e ottenere obbedienza. Si tratta di un argomento forte che egli impiega nella sua battaglia per liberare la religione dall'oscurità dell'ignoranza e riportarla alla verità della ragione, opponendo alla Chiesa della Controriforma, menzognera e violenta, quella dei veri cristiani, coincidente con le comunità antitrinitarie dell'Europa orientale. Lo stesso tema viene ripreso e radicalizzato alcuni anni più tardi nella Disputatio de incertitudine religionis Christianae del 1593, dove si trasforma però in una testimonianza a sostegno della natura storica delle religioni all'interno del percorso di scepsi che conduce Francken fino a mettere in dubbio l'esistenza di Dio. Anche questa idea sembra derivare dal pensiero di Pomponazzi il quale, in particolare nel De incantationibus, parlava dell'uso politico delle religioni e ne individuava la liceità nella funzione di garantire agli uomini leggi conformi alla giustizia. Egli paragonava

${ }^{47}$ C. Francken, op. cit., p. 249.

48 Ibidem, pp. 205-211. 
i contenuti della religione a quelli delle favole dei poeti, in quanto aspirano a una legittimazione razionale ma non hanno alcuna radice metafisica $^{49}$. La religione può risultare utile se serve al legislatore per garantire ordine e benessere alla comunità, ma anche dannosa, come nel caso della demonologia cristiana che rappresenta l'obiettivo polemico dell'opera. Francken recupera l'equivalenza favole-sacre scritture nella Epistola Nicolai Regi, ma la utilizza in chiave aspramente anti cattolica, sostenendo che, senza l'autorità del pontefice che si arroga il diritto di stabilire quali testi sono ispirati dallo spirito santo e quali altri non lo sono, „sacrae literae nihilo plus sint valiturae, quam prophani libri et ipsae Aesopi fabulae" 50 . In realtà, Jószef Simon indica il De incertitudine et vanitate scientiarum et artium di Cornelio Agrippa von Nettesheim come fonte dell'idea della funzione politica della religione presente anche nel De incertitudine religionis Christianae di Francken ${ }^{51}$. Quest'ultimo, però, è lontano dalla sua concezione della natura come un essere vivente dotato di corpo e di anima, quindi soggetto a forze occulte che possono essere dominate attraverso l'uso delle arti magiche, massima espressione della scienza umana e in accordo con la teologia. Non vi sono cenni alla magia in nessuna opera di Francken, che sembra sviluppare piuttosto un materialismo sensistico privo di deroghe. La teoria della conoscenza che viene esposta nel suo ultimo testo a stampa dal titolo Analysis rixae Christianae del 1595 è influenzata ancora una volta dal pensiero di Pomponazzi, ma sembra andare al di là anche dell'orizzonte cosmologico del De incantationibus, che includeva comunque gli influssi astrali e la magia, pur riducendola entro le leggi di natura. Come è noto, Pomponazzi nel De immortalitate animae sosteneva che il pensiero dell'uomo non può prescindere dal corpo, ma ha bisogno di

49 „Nam et in Veteri Lege multa feruntur quae re vera non possunt intelligi ut litera sonat, ut cum dicitur Deum esse allocutum et eius faciem ferri super aquas; sed sunt sensus mystici et dicti propter ignavum vulgum, quod incorporalia capere non potest. Sermo enim Legum, ut inquit Averrois in sua Poesi, est similis sermoni poetarum: nam quamquam poetae fingunt fabulas quae, ut verba sonant, non sunt possibiles, intus tamen continent veritatem", P. Pomponazzi, De incantationibus, p. 110, rr. 331-337. Su questo argomento vedi anche V. Perrone Compagni, Pomponazzi Pietro, in: Il contributo italiano alla storia del pensiero. Filosofia, Roma 2012, pp. 149-157.

50 C. Francken, op. cit., p. 209, rr. 12-13.

${ }^{51}$ Henrici Cornelii Agrippae [- - ] De incertitudine et vanitate scientiarum et artium atque excellentia verbi Dei declamatio, apud Ioannem Petrum in vico Sorbonico, Parisiis 1531. Vedi J. Simon, op. cit., p. 155n. 
rappresentazioni che si fondano sui dati raccolti dai sensi ${ }^{52}$. Anche l'operazione del pensiero, quindi, pur sollevandosi dalla dimensione puramente materiale, non può distaccarsene del tutto perché utilizza i phantasmata, ossia immagini che attingono alla sfera sensibile e hanno pertanto la loro origine nella corporeità. Si trattava di un argomento a sostegno della tesi della mortalità dell'anima, in contrasto con le posizioni di Tommaso d'Aquino. In apertura dell'Analysis rixae Christianae Francken, al fine di spiegare la molteplicità delle religioni e la loro conflittualità, sostiene che il pensiero teologico, anche quando affronta le questioni più astratte, non può evitare che vengano proiettati nella dimensione del divino caratteri che provengono dalla corporeità, perché „nihil corpore vacans in vulgare intrat intellectum; imo ipsis quoque altioribus ingeniis immaterialia cogitantibus ingerit sese corporeum quiddam semper, quod phantasmata solet a philosophis appellari" 53 . Anche in questo caso, dunque, il meccanismo della conoscenza è fondato sul rapporto tra percezioni della realtà sensibile, elaborazione delle immagini che si fondano su di esse, i così detti phantasmata, e loro proiezione nella dimensione degli universali, con l'inevitabile conseguenza che l'attività del pensiero risulta impossibile senza il corpo. La diversità degli ingegni provoca la varietà dei phantasmata e questa è la causa della molteplicità delle religioni e della frammentazione della stessa chiesa cristiana. In realtà Francken non analizza in modo sistematico questa teoria e non ne discute tutte le possibili implicazioni, rendendo difficile procedere a ulteriori congetture circa le fonti. Dopo l'uscita dalla Compagnia di Gesù certamente egli mise alla prova la propria preparazione in una serie di dispute con importanti esponenti del mondo accademico protestante. Prima si misurò a Strasburgo con Johann Marbach sul problema del valore delle opere, poi con Johannes Pappus sulla dottrina dell'ubiquità di Cristo, inerente alla questione della Cena che aveva affrontato in chiave anti-cattolica nel De bestialissima idololatria ${ }^{54}$. Mantenendosi in linea con le posizioni che vi aveva espresso, egli definì

52 Commentando Aristotele, Pomponazzi sostiene per esempio: „non est intelligere absque phantasmate, quod ex experimento comprobatur. Nullo igitur modo intellectus humanus, secundum Aristotelem, habet operationem prorsus a corpore independentem $[--]$. Sic enim verificatur quod semper indiget phantasmate", Petri Pomponatii Mantuani Tractatus de immortalitate animae, [Bononiae] 1534, pp. 12-13.

53 C. Francken, op. cit., p. 287, rr. 24-26.

${ }^{54}$ Vedi: L. Szczucki, Philosophie, pp. 178-180; M. Biagioni, Christian Francken, pp. 60-63. 
l'ubiquità di Cristo „omnium qui unquam in mundo fuerunt, sunt et erunt stultissimum [- ] errorem" $^{25}$. In seguito tenne lezioni sull'Organon di Aristotele presso la famiglia del nobile polacco Giovanni Ostroróg. Infine si diresse a Norimberga e iniziò un corso di filosofia aristotelica presso l'Accademia di Altdorf, che causò lo scontro con il rettore Johannes Thomas Freigius, un convinto sostenitore del pensiero di Pietro Ramo. È difficile misurare l'influenza che queste esperienze esercitarono sulla formazione di Francken il quale, dopo il percorso all'interno dei collegi gesuitici, si affacciava su una realtà molto più vasta e trovava occasioni importanti per allargare le proprie conoscenze. Non solo la lettura e l'analisi di testi, ma anche gli incontri (e gli scontri) con personaggi provenienti da ambienti diversi dovettero influenzare il suo percorso intellettuale. Occasioni di contatto con l'aristotelismo radicale provennero, probabilmente, dal rapporto con l'esule lucchese Simone Simoni, umanista, filosofo, medico, al quale Francken si legò con una forte ma contrastata amicizia.

La mancanza a tutt'oggi di uno studio complessivo su Simoni e, soprattutto, di edizioni critiche delle sue opere rendono complicato il tentativo di tracciarne il percorso intellettuale ${ }^{56}$. Fu uno dei più noti e stimati medici del tempo, tanto che esercitò la professione prima alle dipendenze del principe Augusto di Sassonia a Lipsia per più di dodici anni, dal 1569 al 1581, poi si trasferì a Praga al servizio dell'imperatore Rodolfo II, infine nel 1582 passò a Cracovia ed entrò nel circolo del celebre Crato von Crafftheim, per divenire medico di corte e prendersi cura, insieme con l'altro esule italiano Niccolò Buccella, della salute del re Stefano Báthory. Coloro che lo incontrarono lo descrissero come un uomo dal carattere difficile, molto ambizioso e irascibile, talvolta sprezzante e capace di improvvisi voltafaccia pur di ottenere il successo professionale. Peter Monau, medico anch'egli e amico di Andrea Dudith, Crato von Crafftheim, Thomas Erastus,

55 C. Francken, op. cit., p. 200.

56 Su Simoni vedi soprattutto: D. Cantimori, Un italiano contemporaneo di Bruno a Lipsia, „Studi Germanici”, 1938, pp. 445-466; C. Madonia, Simone Simoni da Lucca, „Rinascimento”, 20, 1980, pp. 161-197; D. Caccamo, Eretici italiani in Moravia, Polonia, Transilvania (1558-1611), Firenze 1999 (2 ed.), pp. 131-145 e passim. Per una bibliografia completa vedi: C. Madonia, Simone Simoni, in: Bibliotheca dissidentium. Repertoire des non-conformistes religieux des seizième et dix-septième siecles, a cura di A. Séguenny, t. 9, Baden-Baden 1988, pp. 25-110. Vedi anche: M. Verdigi, Simone Simoni: filosofo e medico nel '500, Lucca 1997. 
individuava la radice sostanzialmente scientifica e filosofica, quindi aconfessionale, del suo pensiero scrivendo che Simoni non credeva in nessuna religione e per questo poteva essere ritenuto seguace solo di Galeno ${ }^{57}$. In effetti egli mutò religione in funzione della propria convenienza: fu calvinista a Ginevra, luterano a Lipsia, tornò cattolico a Praga e si avvicinò agli antitrinitari a Cracovia, senza esimersi da battute ciniche nei confronti degli esuli italiani religionis causa, ai quali dichiarava, come riferisce il nunzio in Polonia Alberto Bolognetti: „a voi tocca hora d'essere abbrustellati et arrostiti, che il Simonio non è più de vostri" ${ }^{58}$. Simoni aveva compiuto i suoi studi a Padova con maestri quali Vincenzo Maggi e Girolamo Cardano. Ne era uscito con il titolo di dottore in medicina e chirurgia e una formazione imperniata sull'aristotelismo radicale di derivazione averroista, legata alla lezione di Pomponazzi. I suoi interessi teologici, che furono particolarmente vivi nel periodo trascorso a Ginevra e a Basilea, discendevano in realtà da quelli filosofici e si concentravano sul problema del rapporto tra verità di fede e ragione filosofica, affrontato sulla base di un materialismo sensista che rendeva inderogabili i principi della fisica aristotelica. Non stupisce se questo tipo di atteggiamento intellettuale suscitò l'interesse di Francken, che si muoveva su un terreno per molti versi affine. Sappiamo che i due si incontrarono a Vienna verso la fine del 1581 e che si ritrovarono l'anno dopo a Breslavia presso l'umanista Andrea Dudith. Probabilmente affrontarono insieme il viaggio in Polonia, dove giunsero nel 1583. La loro amicizia si concluse con l'aspra polemica intorno alla morte del re Stefano Báthory, alla quale anche Francken prese parte nel 1590 con un'opera rimasta manoscritta ${ }^{59}$. Lech Szczucki fa risalire il loro primo incontro al 1579 a Lipsia ${ }^{60}$, quando ancora Simoni si trovava presso l'elettore Augusto e Francken preparava la pubblicazione del Breve colloquium Iesuiticum. Tale anticipazione consentirebbe di spostare

57 D. Cantimori, Un italiano, p. 459.

${ }^{58}$ Alberto Bolognetti al cardinale Savelli, Cracovia, 23 IV 1583, in: Alberti Bolognetti nuntii apostolici in Polonia Epistolarum et Actorum pars II, ed. E. Kuntze, Cracoviae 1938 (Monumenta Poloniae Vaticana, 6). Vedi anche D. Caccamo, op. cit., p. 139.

59 Si tratta dello Spectrum diurnum Genii Christiani Francken apparens malo Simonis Simonii Genio redatto intorno al 1590 e ora pubblicato in: J. Simon, op. cit., pp. 185-203. In realtà Francken era intervenuto già in precedenza in forma anonima nella Apologia Iohannis Muralti medici contra Simonem Simonium Lucensem del 1589. Vedi C. Francken, op. cit., pp. 273-275.

${ }^{60}$ L. Szczucki, Philosophie, p. 174. 
l'inizio del loro rapporto intellettuale, e quindi anche delle reciproche influenze, alle origini della produzione di Francken. La teoria della dipendenza delle attitudini individuali da caratteri naturali, in relazione alla quale Francken nel Breve colloquium spiega la tendenza degli italiani all'ipocrisia e degli spagnoli all'obbedienza, è posta al centro del De vera nobilitate, pubblicato da Simoni a Lipsia nel 1572. In questo caso egli sostiene il carattere ereditario della nobiltà e della servitù attenendosi allo stesso, rigido naturalismo di derivazione aristotelica che ispirava Francken. Gli uomini nascono fortemente condizionati a svolgere determinati ruoli, non solo per la loro struttura fisica, ma anche per le attitudini intellettuali e morali. Così, afferma Simoni:

ex quali genere canis verbi gratia venaticus suscipiatur ad indagandam fallendamque feram, vel ad eandem persequendam sternendamque; ex quali item equus generatus ad cursus vel ad bellum magni interest; multo magis ex qualibus parentibus homo sit procreatus, ad vitam recte atque ex virtute agendam referre existimabimus ${ }^{61}$.

L'educazione e le circostanze dell'esistenza possono anche andare contro alle attitudini naturali, ma non sono in grado di modificarle profondamente perché il potere della natura è tale („Tanta vis est naturae") ${ }^{62}$ da soverchiare ogni altra forza. Possibili influssi del pensiero di Simoni sono riscontrabili nel De bestialissima idololatria, che Francken pubblicò a Basilea nel 1580. Si tratta, come abbiamo già detto, di uno scritto rivolto contro la dottrina cattolica della transustanziazione, che esaltava fino al paradosso le incongruenze del tentativo concordista di Tommaso d'Aquino e, soprattutto, la loro accettazione acritica da parte degli epigoni scolastici, tra i quali due insegnanti del Collegio romano ben noti a Francken, ossia Diego Paez, docente di teologia scolastica, e Iacobus Gordon, docente di teologia morale ${ }^{63}$. Si trattava di una operazione per certi versi analoga a quella che Simoni aveva condotto contro il teologo luterano e filosofo aristotelico Jakob Schegk di Tubinga sulla dottrina dell'ubiquità di Cristo con una serie di scritti controversistici a partire dalla Declaratio, uscita a Ginevra nel $1566^{64}$, fino all'Antischegkianorum,

${ }^{61}$ Simonis Simoni, De vera nobilitate, Lipsiae 1572, p. 9.

${ }^{6}$ Ibidem, p. 55.

${ }^{63}$ C. Francken, op. cit., pp. 180, 188.

${ }^{64}$ Simoni scrisse l'introduzione della Declaratio eorum quae in libello D.D. Jacobi Scheckii summi et carissimi philosophi de una persona et duabus naturis Christi nonnullis obscuriora et ubiquitati corporis Christi patrocinari sunt visa, apud Johannem 
stampato a Basilea nel $1570^{65}$. Sia Simoni che Francken muovevano dalla verità filosofica per dimostrare l'assurdità della dottrina teologica sul piano della fisica aristotelica: in entrambi i casi veniva irriso il concetto di corporeità immateriale. Francken nel quinto paradosso scriveva che il corpo di Cristo, un uomo di trentatré anni, non può essere compresso nella piccolissima ostia „et inclusus non comprimitur, compressus autem non membris omnibus confuditur, planeque emoritur"66. Un materialismo icastico e diretto, che sembra riecheggiare le parole che Simoni usava, proprio contro la dottrina della transustanziazione, nel De sensuum instrumentis, laddove affermava che ,addit alter in coena Domini ipsissimum Christi corpus et ipsissimum sanguinem cum pane et vino a ministris frangi manibus distribuique: ore autem fidelium sumi et dentibus atteri" ${ }^{3}$. Anch'egli deride le distinzioni scolastiche volte a giustificare razionalmente ciò che non è possibile con acrobatiche invenzioni, del tipo che la carne di Cristo non è onnipresente „non essentialiter et naturaliter, non extensione corporali” ma „ex accidenti, id est per partem omnipraesentem”68. Sempre in quest'opera egli riprende da Aristotele (e da Pomponazzi) una concezione sensistica della conoscenza imperniata sulla funzione dei phantasmata, senza i quali non ritiene possibile alcuna conoscenza ${ }^{69}$. Come abbiamo visto, anche Francken ne fu influenzato, forse proprio grazie al ruolo di collegamento svolto da Simoni. Certamente entrambi svilupparono una gnoseologia contraria a quella platonica, sostenendo l'inscindibilità di forma e materia, di anima e corpo. Nell'Antischegkianorum Simoni afferma che tutte le scienze dipendono dai sensi e per questo „nihil usquam vel unquam concludere volunt, quod cum principiis sensu acquisitis pugnet aut ex iis necessario non sequatur" ${ }^{\prime 70}$ e la nostra conoscenza procede verso gli universali attraverso l'induzione, non accade il contrario: „nam ex sensu sit memoria, ex memoria experimentum, ex experimento procedente confirmatoque

Crispinum, Genevae 1566. Un sintesi della vicenda in: D. Caccamo, op. cit., pp. 131-136; M. Biagioni, Christian Francken, pp. 38-40.

65 Simonis Simonii Lucensis Antischegkianorum liber unus, Basileae 1570.

${ }^{66}$ C. Francken, op. cit., p. 187, rr. 27-29.

67 Simonis Simonii Lucensis, In librum Aristotelis [...] de sensuum instrumentis et de his quae sub sensum cadunt, commentarius unus, excudit Johannes Crispinus, Genevae 1566, p. 89A.

${ }^{68}$ Ibidem.

69 „[- - ] absque phantasmata intelligere non licet”, ibidem, p. 279D.

70 S. Simoni, Antischegkianorum, p. 54. 
universale"71. Per la verità Simoni, almeno formalmente, esclude da questo tipo di percorso la teologia, che invece discende dalla parola di Dio secondo un processo deduttivo ${ }^{72}$. Ma Francken nella disputa con Fausto Sozzini De honore Christi dichiara di voler affrontare le questioni teologiche non in base alle scritture, bensì in base alla sola evidenza razionale ${ }^{73}$, togliendo al pensiero teologico ogni possibilità di deroga dalle leggi della fisica.

La distanza di Francken dal platonismo, come abbiamo visto, emergeva già nel Breve colloquium Iesuiticum in quanto retaggio della formazione filosofica acquisita presso il Collegio romano. Del resto, solo all'interno di un sistema improntato a un rigoroso naturalismo aristotelico poteva assumere significato una concezione della conoscenza fondata sul processo induttivo, anche per quanto riguarda il pensiero religioso, e trovava quindi spiegazione lo sgomento dinanzi alla scoperta delle affinità tra le pratiche religiose dei giapponesi e quelle dei gesuiti che aveva precipitato Francken „ex astris in terram" ${ }^{74}$, come scriveva egli stesso. La prima traccia di una fonte platonica negli scritti di Francken è contenuta nella Praecipuarum enumeratio causarum del 1584, dove egli ricorre all'esempio degli argomenti utilizzati dal filosofo neoplatonico Proclo per sostenere l'eternità del mondo in polemica con i cristiani. In realtà si tratta di una indicazione che solo indirettamente riconduce al testo del $D e$ aeternitate mundi contra Proclum di Giovanni Filopono. La fonte alla quale Francken attinge con precise citazioni è invece il trattato del gesuita Benedictus Pereira dal titolo De communibus omnium rerum naturalium principiis et affectionibus nel quale vengono discussi, attraverso il testo di Filopono, i Viginti argumenta Procli pro aeternitate mundi $i^{75}$. Si tratta di un dato comunque interessante perché Pereira, che sappiamo essere stato docente al Collegio romano e che Francken quindi conobbe, suscitò polemiche interne proprio per la sua apertura al platonismo. Agli anni della prima formazione (dal 1570 al 1575 circa) sembrano risalire, pertanto, i contatti di Francken con il platonismo, sebbene filtrati attraverso l'ottica controversistica di Pereira. Ma nella Praecipuarum enumeratio causarum, che pure risulta un testo organicamente strutturato su coordinate aristotelico-averroiste, oltre

${ }^{71}$ Ibidem, p. 56.

72 Ibidem, p. 54.

${ }^{73}$ C. Francken, op. cit., p. 213, rr. 14-16.

${ }^{74}$ Ibidem, p. 171, rr. 4-5.

${ }^{75}$ Ibidem, p. 249. 
al richiamo a Proclo sono rilevabili anche sporadici segnali di una sensibilità diversa nei confronti di concetti fondamentali come quelli di natura e di ragione. Szczucki segnala l'affinità di alcuni passaggi della Praecipuarum enumeratio causarum con il commento di Ficino al Timeo, che forse sono il risultato di echi giunti a Francken attraverso gli scritti dell'umanista e antitrinitario Johannes Sommer, anch'egli di origine sassone ed emigrato in Transilvania ${ }^{76}$. In particolare risultano inusuali rispetto alle precedenti opere di Francken le immagini della ragione naturale come luce che penetra nell'universo („humanae rationis naturale lumen divinis in rebus effulgens”), utilizzata anche in chiave metaforica per sottolineare la conformità dei misteri divini alle leggi di natura („religionis nostrae mysteria non esse contra rationem [- ] sed tam consentanea rationi quam parva veritas magnae et exiguum lumen maximo est simile") ${ }^{77}$. La figura della luce della ragione che diffonde la verità („micante rationis scintillula”, "lumen ingenii") ${ }^{78}$ viene posta in contrasto con l'autorità della Chiesa romana che vi si oppone con la minaccia delle pene e l'allettamento degli onori. Si tratta di figure comuni nel linguaggio di qualunque generica forma di platonismo, ma che alludono all'idea di una manifestazione naturale di Dio nella natura fino ad allora estranea alla riflessione di Francken. Può rappresentare un dato interessante il fatto che Francken con ogni probabilità incontrò già a Cracovia alla fine del 1583 l'esule fiorentino Francesco Pucci ${ }^{79}$, al quale lo legò una duratura amicizia, tanto che i due si ritrovarono a Praga nell'estate del $1587^{80}$. Pucci si era recato in Polonia per continuare con Fausto

${ }^{76}$ L. Szczucki, Philosophie, p. 197.

77 C. Francken, op. cit., p. 248, rr. 7, 21-24.

78 Ibidem, rr. 24, 28.

79 Su Francesco Pucci sono fondamentali le ricerche di D. Cantimori, L. Firpo e A. Rotondò. Per una bibliografia aggiornata rimando però ai due studi più recenti su di lui, ossia: G. Caravale, Il profeta disarmato. L'eresia di Francesco Pucci nell'Europa del Cinquecento, Bologna 2011 (ora anche in inglese: The Italian Reformation outside Italy. Francesco Pucci's Heresy in Sixteenth-Century Europe, Leiden-Boston 2015); M. Biagioni, Francesco Pucci e l'Informatione della religione cristiana, Torino 2011.

${ }^{80}$ Il 9 VII 1587 si recarono insieme da Praga alla residenza del nobile boemo Wilem Rožmberk a Třeboň per fare visita all'alchimista inglese John Dee e al veggente Edward Kelley che risiedevano presso di lui. La notizia è contenuta in: The private Diary of dr. J. Dee and the Catalogue of his library, edi. by J. Orchard Halliwell, London 1842, p. 23. Pubblicata anche in: F. Pucci, Lettere, documenti e testimonianze, vol. 2, a cura di L. Firpo, R. Piattoli, Firenze 1959, p. 202. Sulla vicenda vedi M. Biagioni, Christian Francken, pp. 122-127. 
Sozzini il dibattito sulla natura immortale di Adamo, iniziato a Basilea nel $1577^{81}$, e poneva al centro della sua entusiastica predicazione proprio l'idea della conoscenza naturale di Dio che, attraverso la sua sapienza e ragione (il logos) risplendente nel creato, fornisce a tutti gli uomini, nessuno escluso, al di fuori delle chiese e della rivelazione, la possibilità di ottenere la salvezza. Si tratta di principi esposti nel volume dal titolo Informatione della religione Christiana da lui pubblicato a Londra alla fine del $1579^{82}$ e una copia del quale era giunta nelle mani del Sozzini a Cracovia ${ }^{83}$. Vi abbondano le immagini del logos come „sapienza et ragione, la quale penetra e risplende per tutto l'universo e in noi stessi con divinissimi raggi”, come "primo unico lume ch'è prodotto da quello eterno lume”, come „splendentissimo lampo di Dio del cielo e della terra, che illumina ogni uomo che viene in questo mondo" ${ }^{4}$. La luce della ragione è il fondamento della religione naturale e il presupposto per interpretare le scritture, che altrimenti risultano un inestricabile labirinto. Se l'uomo non si dispone a riceverla, la rivelazione attraverso la parola rimane lettera morta. Per questo Pucci preparò nel 1583 delle theses de fide e le discusse in via preliminare con Sozzini prima di iniziare il dibattito testuale sulla natura di Adamo ${ }^{85}$. Egli vi affermava che la ragione naturale è il presupposto necessario „ad perpendenda ordine et ratione scripta prophetarum et apostolorum, eaque commode intelligenda et interpretanda": gli interpreti delle scritture che ne prescindono non possono che litigare tra di loro e travisarne il significato, come accade sempre più spesso $^{86}$. Pucci si rivolgeva così ai fratres della Ecclesia minor, arbitri

${ }^{81}$ A proposito del dibattito tra Pucci e Fausto Sozzini sulla natura immortale di Adamo vedi: M. Biagioni, La ragione dell'immortalità: la disputa tra Francesco Pucci e Fausto Sozzini "de statu primi hominis ante lapsum”, in: Faustus Socinus and his Heritage, ed. by L. Szczucki, Kraków 2005, pp. 53-89; idem, Adamo nell'età moderna, in: F. Sozzini e F. Pucci, De statu primi hominis ante lapsum disputatio, a cura di M. Biagioni, Roma 2010, pp. IX-LXIV.

82 Ora in: M. Biagioni, Francesco Pucci, pp. 87-130.

${ }^{83}$ Lettera di Fausto Sozzini ad Andrea Dudith, Cracovia, 3 XII 1580, in: Fausti Socini Senensis op. cit., vol. 1, p. 497 (anche in: F. Pucci, Lettere, II, p. 147).

${ }^{84}$ M. Biagioni, Francesco Pucci, p. 91.

85 Ora pubblicate, con erronea datazione e attribuzione, in: M. Biagioni, Universalismo e tolleranza nel pensiero di Francesco Pucci, in: La formazione storica della alterità. Studi di storia della tolleranza nell'età moderna offerti a Antonio Rotondò, t. 1, Firenze 2001, pp. 357-360. Per l'esatta datazione vedi idem, La ragione, p. 58.

${ }^{86}$ M. Biagioni, Universalismo, p. 360. 
della sua disputa con Sozzini, affinché „naturalibus rationibus potius quam scripto Dei verbo agendum esset" ${ }^{27}$. Francken, come abbiamo detto, avanzò una richiesta analoga in apertura della disputa De honore Christi, ponendo come pregiudiziale che avrebbe discusso non „sacrae Scripturae testimoniis, quae detorqueri possunt, atque adeo ab omnibus nunc, etiam absurdissimis sectariis, detorquentur ad victoriam, sed agam ratione" 88 . Si tratta di punti di contatto contenutistici e anche linguistici, non supportati, però, da testimonianze esplicite che dissipino dubbi circa l'ipotesi, per quanto circostanziata, di un influsso di Pucci. Certamente il pensiero di Pucci si collocava entro le coordinate del neoplatonismo fiorentino che, come scrive Giorgio Caravale, ne aveva caratterizzato la formazione fino dai primi anni presso l'Accademia dei Lucidi con la frequentazione dei testi di Marsilio Ficino e di Giovanni Pico della Mirandola ${ }^{89}$. Anche Francken si avvicinò a questi autori, senza però che essi modificassero realmente la propria Weltanschauung. Pico della Mirandola compare nella sua opera solo con un testo che viene citato due volte. Si tratta di un brano tratto dalla lettera indirizzata al nipote Gian Francesco, che compare già nella Epistola Christiani Francken, in qua deplorat suum a Societate Iesu et Ecclesia Catholica discessum composta nel $1581^{90}$. Esso viene poi ripreso otto anni dopo nell'Apologia Iohannis Muralti ${ }^{91}$, senza sostanziali modifiche e con uno scopo simile, cioè portare l'esempio di un dotto umanista che sostiene la ragionevolezza della verità evangelica e soprattutto esalta il messaggio morale dell'insegnamento di Cristo, con una critica neppure tanto velata al comportamento dei fedeli e degli uomini di chiesa ${ }^{92}$. Ma è una citazione che appare poco incisiva sul piano gnoseologico, nel senso che non induce a ipotizzare una reale apertura dell'autore verso il pensiero del Neoplatonismo fiorentino. Più estesa, ma non molto diversa qualitativamente, è la presenza di Marsilio Ficino, sebbene in questo caso la prima attestazione certa risalga solo alla seconda metà del 1588 , quando Francken risedette per alcuni mesi presso la dimora del nobile Miklós Szokoli a Kisvárda in Ungheria e attinse ai volumi della sua

\footnotetext{
${ }^{87}$ Fausti Socini Senensis op. cit., vol. 1, p. 379.

${ }^{88}$ C. Francken, op. cit., p. 213, rr. 14-16.

${ }^{89}$ G. Caravale, Il profeta, pp. 44-48, 60-67.

${ }^{90}$ C. Francken, op. cit., p. 202.

${ }^{91}$ Ibidem, p. 274.

92 „Sed longe maior insania, si de evangelii veritate non dubitas, vivere tamen quasi de eius falsitate non dubitares", ibidem, rr. 8-9.
} 
ricca biblioteca, trovandovi certamente opere ficiniane. Lo dichiara egli stesso nello Spectrum diurnum genii Christiani Francken, il testo manoscritto con il quale prese parte alla polemica tra Simone Simoni e Niccolò Buccella in seguito alla morte del loro comune assistito, il re Stefano Báthory ${ }^{93}$. Nella lettera dedicatoria a Sigismondo Báthory egli riporta un pensiero che, come sottolinea József Simon, è attinto dalla epistola di Ficino a Giovanni Pico della Mirandola del 30 maggio 1488, laddove riflette sulle vicissitudini della vita che spesso affliggono i grandi uomini ${ }^{94}$. Ma neppure questa è una citazione alla quale sembra possibile attribuire uno spessore speculativo, piuttosto un omaggio reso a un autore che viene volutamente posto in rilievo. $\mathrm{Ha}$ un carattere simile il richiamo immediatamente successivo, sempre dalle epistole, ai rischi che derivano al principe dall'odio e dall'invidia dei sudditi ${ }^{95}$. Più interessante è il brano tratto dalla Theologia Platonica che compare nella parte conclusiva dell'opera, nel quale Ficino afferma che la fragilità e l'incostanza della fede, spesso caratteristica degli uomini dotti, è dovuta all'abitudine di indagare razionalmente le cause dei fenomeni: una curiositas che confligge con la necessità di accettare per fede i misteri della religione ${ }^{96}$. Si tratta di un argomento che Francken riprende dall'Apologia Iohannis Muralti medici contra Simonem Simonium Lucensem del 1589, dove era accompagnato dalla definizione di Ficino come „vir ingeniosissimus et maxime pius"97. Ancora alla Theologia Platonica conducono due nessi concettuali che József Simon individua nella Disputatio de incertitudine religionis Christianae, entrambi focalizzati sulla dimostrazione dell'esistenza di Dio in funzione della mole del mondo ${ }^{98}$. Sono idee, però, che nella dinamica del dialogo vengono esposte dal teologo e confutate dal filosofo, quindi in posizione debole sul piano argomentativo. La presenza di questi segnali, insomma, fornisce tutt'al più una testimonianza di generiche diversioni rispetto al naturalismo aristotelico che continuava a costituire la struttura portante della sua riflessione. Più puntuali e diffuse, come abbiamo visto, appaiono le ricorrenze di espressioni e di idee tratte dal linguaggio e dal pensiero di Pucci, rispetto alle quali le citazioni di Ficino appaiono come un corollario.

\footnotetext{
93 J. Simon, op. cit., p. 190.

${ }^{94}$ Ibidem, pp. 185-186.

95 Ibidem, p. 189.

96 Ibidem, pp. 200-201.

97 C. Francken, op. cit., p. 275.

98 J. Simon, op. cit., pp. 176, 178.
} 
È difficile stabilire quanto il rapporto con l'esule fiorentino abbia esercitato suggestioni sulla riflessione di un uomo come Francken, esposto a crisi profonde e soggetto per fragilità di carattere a continua inquietudine. Ma non è da escludere che sia da far risalire alla sua frequentazione l'insorgenza di sfumature neoplatoniche negli scritti successivi al 1583.

Si tratta comunque di tracce da non sottovalutare nella prospettiva di una crisi delle certezze che prese campo nel pensiero di Francken forse già a partire dal primo soggiorno a Cluj in Transilvania nel 1584, dopo la disputa con Sozzini e la stesura della Praecipuarum enumeratio causarum. In quell'opera culminava il suo tentativo di trovare una religione razionale, seguito a breve dalla disillusione circa la possibilità di far coincidere ragione filosofica e fede, che si accompagnava a nuove ansie. Nell'epistola introduttiva al commento del pastore luterano Thomas Kirchmair al Manuale di Epitteto, del quale Francken curò la pubblicazione l'anno successivo, egli mostra ancora interesse verso la possibilità di trarre insegnamenti utili dall'incontro di un filosofo antico (Epitteto) e un commentatore cristiano (Kirchmair) per illuminare la via che conduce alla felicità ${ }^{99}$. Ma sono gli ostacoli che vi si frappongono, in particolare gli appetiti che gli uomini condividono con le bestie, ad avere il sopravvento, rendendo illusoria questa possibilità. Dalla fine del 1588, dopo il dibattito a Cracovia con il frate minorita Moro Saraceno di Fossombrone sulle cause che alimentano i conflitti tra i cristiani e in seguito alla frequentazione della biblioteca del nobile Miklós Szokoli a Kisvárda, negli scritti di Francken cominciano a comparire indizi della lettura delle opere di Sesto Empirico, con una conseguente apertura a posizione scettiche. Come è noto la circolazione di Sesto in età moderna fu determinata soprattutto dalla pubblicazione dell'edizione latina degli Hypotyposeon libri a Parigi nel 1562 per opera di Henri Estienne, alla quale fece seguito quella dell'Adversus mathematicos ad Anversa e a Parigi nel 1569 per opera di Gentien Hervet. Francken probabilmente conosceva lo scetticismo accademico fino dagli studi al Collegio romano attraverso l'opera di Cicerone, la cui presenza è attestata già nel Breve colloquium Iesuiticum con riferimenti alle Tusculanae disputationes e al De natura deorum $^{100}$. La lettura di Sesto, che gli apriva le porte allo scetticismo pirroniano, dovette però impressionarlo a tal punto che la struttura

${ }^{99}$ C. Francken, op. cit., pp. 103-104.

100 Ibidem, pp. 168, 175-176. 
argomentativa del dibattito sull'esistenza di Dio nella Disputatio de incertitudine religionis Christianae, della quale una prima versione circolava fino dall'inizio degli anni Novanta ${ }^{101}$, risulta ispirata proprio all'Adversus mathematicos. József Simon ne ha segnalato citazioni nell'argomento trentasei e nella breve antologia di pensieri di filosofi antichi che chiude l'opera ${ }^{102}$. Ma l'influenza dell'opera di Sesto è assai più estesa e profonda. Francken riprende infatti dall'Adversus mathematicos i contenuti del capitolo An sint Dii nel quale Sesto afferma che coloro i quali vogliono dimostrare l'esistenza degli Dei lo fanno utilizzando quattro tipi di argomenti, che sono, nell'ordine: il „consensus omnium hominum”, l',ordo mundi et distributio”, gli „absurda quae consequuntur iis qui Deum tollunt", l', ablatio rationum quae obiiciuntur"103. Dall'argomento ventuno, che apre la parte conclusiva del dialogo composta da diciassette interventi del teologo e altrettanti del filosofo sul problema dell'esistenza di Dio o quanto meno della sua dimostrabilità, sino alla fine dell'opera ${ }^{104}$, viene riproposto lo stesso schema con numerosi richiami a Sesto, più o meno espliciti. La sola modifica è costituita dall'omissione dell'ultimo passaggio, ossia la rimozione delle ragioni che negano l'esistenza di Dio, imposta dalla stessa natura del dialogo, durante il quale il filosofo agisce invece per lo scopo opposto di rimuovere le ragioni che la proverebbero. Questa sezione è sostituita da una piccola antologia di opinioni di filosofi antichi sull'ateismo, anch'esse presenti per la maggior parte nell'Adversus mathematicos ${ }^{105}$. La presenza di Sesto nel pensiero di Francken durante gli anni trascorsi in Transilvania e in Boemia coincide con una vera e propria svolta. La Disputatio de incertitudine religionis Christianae riproduce il dibattito sostenuto contro Moro Saraceno di Fossombrone che si svolse probabilmente nella prima metà del 1588 a Cracovia. Teologo francescano e filosofo aristotelico, Moro Saraceno sosteneva che solo la cattiva interpretazione delle scritture

${ }^{101}$ Vedi B. Keserü, Christian Franckens Tätigkeit im ungarischen Sprachgebiet und sein unbekanntes Werk "Disputatio de incertitudine religionis Christianae”, in: Antitrinitarianism in the Second Half of the $16^{\text {th }}$ Century, ed. by R. Dán, A. Pirnát, Budapest 1982, pp. 73-84. Vedi anche M. Biagioni, Christian Francken, pp. $113-115$.

102 J. Simon, op. cit., pp. 179-181.

${ }^{103}$ Sexti Empirici Adversus mathematicos, hoc est adversus eos qui profitentur disciplinas, Gentiano Herveto Aurelio interprete, ex officina Christophori Plantini, Antverpiae 1569, p. 265.

104 J. Simon, op. cit., pp. 171-181.

105 Sexti Empirici op. cit., pp. 264-265. 
fosse la causa dei conflitti tra cristiani. In sostanza, egli fondava la sua posizione sul principio che il testo sacro è inoppugnabile se messo a fuoco secondo le dieci categorie aristoteliche che definiscono l'essere $^{106}$. Francken, anch'egli battagliero sostenitore del pensiero aristotelico, diversamente dal solito non si sentì in linea con una dottrina che comunque si collocava entro quello stesso orizzonte culturale. Pur manifestando la massima stima per l'avversario, definito „doctissimum doctorem theologum" 107 , e pur avendo negli anni precedenti creduto fermamente in una religione razionale, adesso invece individuava la causa delle controversie tra cristiani nel fatto che la religione „nulla tamen certa posse ratione demonstrari”, precisando che „certam autem rationem dico, quae non possit infringi, sed cogat quodammodo, etiam nolentem et invitum, ad assentiendum; cuiusmodi est arithmetica [- ] ratio" 108 . Nel testo che Francken compose ispirandosi a questo dibattito, si percepisce che la voce del teologo e quella del filosofo non riproducono semplicemente il pensiero di Moro, la prima, quello di Francken, l'altra, ma delineano una relazione più complessa e travagliata. Sono comunque le parole del filosofo a proporre in modo più organico la posizione maturata da Francken in quegli anni. I suoi interventi tracciano un vero e proprio percorso di scepsi che conduce all'epochè, in coerenza con la posizione di Sesto che riteneva impossibile stabilire se gli Dei esistono o non esistono „per parem vim adversantium orationum”109 e per questo considerava più saggio accettare la religione degli avi, insieme ai costumi e alle leggi, senza esporsi sul problema della effettiva corrispondenza di essa con la verità ${ }^{110}$. Simile sarebbe stato l'atteggiamento di Francken negli ultimi anni. Giunto a dubitare con la ragione dell'esistenza di Dio, preferì ritrarsi dal vuoto che l'ateismo sembrava spalancargli dinanzi e sottomettersi all'autorità della Chiesa di Roma, anche a costo di subire il processo e la condanna al carcere a vita presso il Santo Uffizio a Roma. Ma il suo scetticismo, diversamente dallo scetticismo cristiano cinquecentesco, rivelava una forza corrosiva che metteva in questione la stessa nozione di Dio. József Simon sottolinea alcune affinità, anche nel titolo, tra la Disputatio de

${ }^{106}$ La sua posizione è sintetizzata da Francken in: Analysis rixae Christianae (Ch. Francken, op. cit., p. 291).

107 Ibidem.

${ }^{108}$ Ibidem, p. 289, rr. 14, 18-20.

109 Sexti Empirici op. cit., p. 265.

110 Ibidem, pp. 263-264. 
incertitudine religionis Christianae di Francken e la De incertitudine et vanitate scientiarum et artium di Agrippa von Nettesheim ${ }^{111}$. La svalutazione di ogni forma di conoscenza che conduce Agrippa all'esaltazione della fede come unica via verso la verità non coincide però con gli esiti del percorso di Francken il quale, solo dopo avere verificato che la ragione costringe a dubitare dell'esistenza di Dio, accetta di rinunciare alla ragione pur di salvare la nozione di Dio. Nelle ultime opere di Francken, compaiono anche richiami ad Erasmo ${ }^{112}$. Ma neppure lo scetticismo erasmiano, che nasceva dall'avversione nei confronti del razionalismo teologico a favore dell'imitazione di Cristo e della semplificazione dottrinale, ha molto in comune con quello di Francken, perché nel primo caso rimaneva comunque indiscusso il postulato dell'esistenza di Dio, nell'altro invece il dubbio sistematico finiva per erodere anche quel fondamentale presupposto. Per questi motivi, a mio avviso, il caso di Christian Francken presenta caratteristiche del tutto peculiari nel panorama culturale del tardo Cinquecento che consentono di collocarlo alle origini del dibattito che, nel corso dell'età moderna, ha condotto al riconoscimento della dignità intellettuale dell'ateismo, precedentemente considerato un paradigma di pura negatività, un paradosso talmente infamante da implicare l'esclusione dal consorzio civile.

\section{Bibliografia}

Henrici Cornelii Agrippae [- - De incertitudine et vanitate scientiarum et artium atque excellentia verbi Dei declamatio, apud Ioannem Petrum in vico Sorbonico, Parisiis 1531

M. Biagioni, La critica all'argomento del "consensus gentium”, in: Le ragioni degli altri, a cura di M. Priarolo, E. Scribano, Philosophica 3, Edizioni Ca' Foscari Digital Publishing, febbraio 2017, http://edizionicafoscari. unive.it/it/edizioni/collane/philosophica/, pp. 47-62

M. Biagioni, Francesco Pucci e l'Informatione della religione cristiana, Torino 2011

M. Biagioni, La ragione dell'immortalità: la disputa tra Francesco Pucci e Fausto Sozzini "de statu primi hominis ante lapsum”, in: Faustus Socinus and his Heritage, ed. by L. Szczucki, Kraków 2005, pp. 53-89.

${ }^{111}$ J. Simon, op. cit., pp. 82, 138.

112 Nelle Mirabilium visionum del 1589 e nel Dolium Diogenis del 1595 (C. Francken, op. cit., pp. 269, 280, 283). J. Simon (idem, op. cit., pp. 195-196) individua una citazione anche nello Spectrum. 
M. Biagioni, The Radical Reformation and the Making of Modern Europe. A Lasting Heritage, Leiden-Boston 2017.

M. Biagioni, Universalismo e tolleranza nel pensiero di Francesco Pucci. La formazione storica della alterità. Studi di storia della tolleranza nell'età moderna offerti a Antonio Rotondò, vol. 1, Firenze 2001, pp. 331-360

Alberti Bolognetti nuntii apostolici in Polonia Epistolarum et Actorum pars II, ed. E. Kuntze, Cracoviae 1938 (Monumenta Poloniae Vaticana, 6)

D. Caccamo, Eretici italiani in Moravia, Polonia, Transilvania (1558-1611), Firenze 1999

T. Campanella, Quod reminiscentur et convertentur ad dominum universi fines terrae, a cura di R. Amerio, Padova 1939.

D. Cantimori, Un italiano contemporaneo di Bruno a Lipsia, „Studi Germanici”, 1938, pp. 445-466

D. Cantimori, Eretici italiani del Cinquecento e prospettive di storia ereticale italiana del Cinquecento, a cura di A. Prosperi, Torino 1992

G. Caravale, Il profeta disarmato. L'eresia di Francesco Pucci nell'Europa del Cinquecento, Bologna 2011

G. Caravale, The Italian Reformation outside Italy. Francesco Pucci's Heresy in Sixteenth-Century Europe, Leiden-Boston 2015

Ch. Clavius Bambergensis, Euclidis elementorum libri $X V$, apud Vincentium Accoltum, Romae 1574

M. da Costa, Rerum e Societate Iesu in Oriente gestarum volumen, Calenius et Quentel, Colonia 1574

L. Firpo, Scritti sulla Riforma in Italia, Napoli 1996

M. Firpo, Juan de Valdés and the Italian Reformation, Ashgate 2015

M. Firpo, Juan de Valdés e la Riforma nell'Italia del Cinquecento, Roma-Bari 2016

Ch. Francken, Opere a stampa, a cura di M. Biagioni, premessa di G. Paganini, Roma 2014

R. Gatto, Cristoforo Clavio, in: Enciclopedia Italiana di Scienze, Lettere ed Arti. Contributo italiano alla storia del pensiero. Ottava appendice, Roma 2013, pp. 236-240

B. Keserü, Christian Franckens Tätigkeit im ungarischen Sprachgebiet und sein unbekanntes Werk "Disputatio de incertitudine religionis Christianae”, in: Antitrinitarianism in the Second Half of the $16^{\text {th }}$ Century, ed. by R. Dán, A. Pirnát, Budapest 1982, pp. 73-84

C. Madonia, Simone Simoni da Lucca, „Rinascimento”, 20, 1980, pp. 161-197

C. Madonia, Simone Simoni, in: Bibliotheca dissidentium. Repertoire des non-conformistes religieux des seizième et dix-septième siecles, a cura di A. Séguenny, t. 9, Baden-Baden 1988, pp. 25-110

G. P. Maffei, De vita et moribus Ignatii Loyolae, qui Societatem Iesu fundavit, libri III, apud Franciscum Zannettum, Romae 1585 
Benedicti Pererii Societatis Iesu De communibus omnium rerum naturalium principiis et affectionibus libri quindecim qui plurimum conferunt ad eos octo libros Aristotelis qui de physico auditu inscribuntur intelligendos, apud Franciscum Zanettum et Bartholomaeum Tosium, Romae 1576

V. Perrone Compagni, Pomponazzi Pietro, in: Il contributo italiano alla storia del pensiero. Filosofia, Roma 2012, pp. 149-157.

Petri Pomponatii Mantuani Tractatus de immortalitate animae, [Bononiae] 1534

Petri Pomponatii Mantuani Libri quinque de fato, de libero arbitrio et de praedestinatione, III, 14, a cura di R. Lemay, Padova 1957

Pietro Pomponazzi, De incantationibus, a cura di V. Perrone Compagni, con la collaborazione codicologica di L. Regnicoli, Firenze 2011

F. Pucci, Lettere, documenti e testimonianze, vol. 1-2, a cura di L. Firpo, R. Piattoli, Firenze 1955-1959

A. Rotondò, Alcune valutazioni su "Eretici italiani del Cinquecento”, „Studi Storici”, 34/4, 1993/1994, pp. 769-775

A. Rotondò, Studi di storia ereticale del Cinquecento, vol. 1-2, Firenze 2008

J. D. Roth, Recent Currents in the Historiography of the Radical Reformation, „Church History”, 71/3, 2002, pp. 523-535

Sexti Empirici Adversus mathematicos, hoc est adversus eos qui profitentur disciplinas, Gentiano Herveto Aurelio interprete, ex officina Christophori Plantini, Antverpiae 1569

Simonis Simonii Declaratio eorum quae in libello D.D. Jacobi Scheckii summi et carissimi philosophi de una persona et duabus naturis Christi nonnullis obscuriora et ubiquitati corporis Christi patrocinari sunt visa, apud Johannem Crispinum, Genevae 1566

Simonis Simonii Lucensis, In librum Aristotelis [...] de sensuum instrumentis et de his quae sub sensum cadunt, commentarius unus, excudit Johannes Crispinus, Genevae 1566

Simonis Simonii Lucensis Antischegkianorum liber unus, Basileae 1570

J. Simon, Die Religionsphilosophie Christian Franckens 1552-1610? Atheismus und radikale Reformation in Frühneuzeitlichen Ostmitteleuropa, Wiesbaden 2008

Fausti Socini Senensis Opera Omnia in duos tomos distincta, vol. 1-2, Irenopoli [Amsterdam] 1656 (reprint a cura di E. Scribano, Siena 2004)

Fausto Sozzini e Francesco Pucci, De statu primi hominis ante lapsum disputatio, a cura di M. Biagioni, Roma 2010

L. Szczucki, Philosophie und Autorität. Der Fall Christian Francken, in: Reformation und Frühaufklärung in Polen. Studien über den Sozinianismus und seine Einfluß auf das westeuropäische Denken im 17. Jahrhundert, hrsg. von P. Wrzecionko, Gottingen 1977, pp. 157-243

L. Szczucki, W kręgu myślicieli heretyckich, Wrocław 1972 
M. Verdigi, Simone Simoni: filosofo e medico nel '500, Lucca 1997

R. G. Villoslada, Storia del collegio romano dal suo inizio (1551) alla soppressione della Compagnia di Gesù (1773), apud aedes Universitatis Gregorianae, Romae 1954

J. Wijaczka, Christian Francken, in: Bibliotheca dissidentium. Repertoire des non-conformistes religieux des seizième et dix-septième siècles, a cura di A. Séguenny, t. 12, Baden-Baden 1991

G. H. Williams, The Radical Reformation, Kirksville 2000

\section{Humanist Sources of Christian Francken's Thought}

Christian Francken was one of the most interesting radical reformers in the sixteenth century. This essay tries to trace some of the main sources of his thought by a textual analysis of the works. He was deeply influenced by Pietro Pomponazzi and, later, by Sextus Empiricus. Much less important was the influence of Neoplatonism, even if Francken knew the thought of Pico della Mirandola and Marsilio Ficino. Pomponazzi's works, above all De incantationibus, De immortalitate animae and De fato, libero arbitrio et praedestinatione, were some of the main sources in early theological and philosophical writings by Francken, from the Breve colloquium Iesuiticum (1579) to the Praecipuarum enumeratio causarum (1584), when he still believed in a reasonable religion. He became acquainted with Sextus Empiricus' works probably around 1587: important quotations from the Adversus mathematicos by Sextus appear in the Disputatio inter theologum et philosophum de incertitudine religionis Christianae, written by Francken in the following years and possibly finished in Cluj in 1593. From then on he seemed more and more close to radical skepticism, so that he no longer believed in a rational religion, nor that the existence of God were demonstrable. He was not too far from unbelief, before submitting at last to Roman Church's authority.

Mario Biagioni jest niezależnym badaczem i nauczycielem łaciny oraz literatury włoskiej w szkole średniej. Mieszka w Pistoi (Włochy) i współpracuje z prof. Emanuelą Scribano (Università degli Studi Ca' Foscari, Wenecja) oraz Lucią Felici (Università degli Studi di Firenze), koncentrując swoje zainteresowania badawcze na związkach pomiędzy radykalną reformacją a kształtowaniem się współczesności. Jest autorem wielu monografii i artykułów na temat radykalnej reformacji, m.in. Christian Francken e la crisi intellettuale della Riforma („Storia e Letteratura”, 2014) oraz The Radical Reformation and the Making of Modern Europe. A Lasting Heritage (Brill, 2017). 
Mario Biagioni is an independent scholar and teacher of Latin and Italian Literature at an Italian secondary school. He lives in Pistoia (Italy) and collaborates with professors Emanuela Scribano (Università degli Studi Ca' Foscari, Venezia) and Lucia Felici (Università degli Studi di Firenze), focusing his research interests on the relationships between the Radical Reformation and the making of modernity. He has published several monographs and articles on the Radical Reformation, including Christian Francken e la crisi intellettuale della Riforma (Storia e Letteratura, 2014) and The Radical Reformation and the Making of Modern Europe. A Lasting Heritage (Brill, 2017).

E-mail: mario.biagioni9@tin.it 